\title{
Impacts of a National Action Plan on Antimicrobial Use in China: A Multi-Center Retrospective Study
}

\author{
Lidao Bao ${ }^{1, \dagger}$, Gaowa Sharen ${ }^{2, \dagger}$, Sha $\mathrm{Li}^{1},{\text { Xianhua } \operatorname{Ren}^{1} \text {, Ruilian Ma }}^{1, *}$ \\ ${ }^{1}$ Department of Pharmacy, Affiliated Hospital of Inner Mongolian Medical University, Hohhot, P. R. China \\ ${ }^{2}$ Department of Ultrasound, Affiliated Hospital of Inner Mongolian Medical University, Hohhot, P. R. China
}

Email address:

maruilian2017@163.com(Ruilian Ma)

*Corresponding author

$\dagger$ Lidao Bao and Gaowa are co-first authors

\section{To cite this article:}

Lidao Bao, Gaowa Sharen, Sha Li, Xianhua Ren, Ruilian Ma. Impacts of a National Action Plan on Antimicrobial Use in China: A Multi-Center Retrospective Study. Science Journal of Public Health. Vol. 6, No. 3, 2018, pp. 75-81. doi: 10.11648/j.sjph.20180603.12

Received: April 10, 2018; Accepted: April 24, 2018; Published: May 10, 2018

\begin{abstract}
This study retrospectively analyzed antimicrobial prescription and concomitant economic outcomes in 28 Chinese state-owned hospitals before and after the intervention introduced by the Action Plan. The outcomes of national intervention were evaluated by analyzing statistics collected before and after the Action Plan which came into effect as of July 1, 2011. The fourth quarters of 2010 and 2011 were set as the baseline and intervention periods, respectively. The percentage of patients receiving antimicrobial prescription, the intensity of antimicrobials prescribed, economic indexes related to antimicrobial prescribing, and antimicrobial prophylaxis in clean surgical procedures were investigated, respectively. First, during the intervention period, the percentage of patients receiving antimicrobial treatment dropped to $15.6 \%$ and $49.1 \%$ in outpatient and inpatient settings, respectively. The intensity of antimicrobial prescribed decreased to 50.29 DDD (defined daily dose) in outpatient setting. The defined daily doses (DDDs) per thousand patients per day decreased to 12914.33 DDDs in outpatient settings. The results were statistically significant as compared to those in the baseline period $(\mathrm{P}<0.001)$. Second, the expenses on antimicrobials significantly decreased $(\mathrm{P}<0.001)$. Finally, the percentage of patients receiving antimicrobial prophylaxis for clean surgical procedures decreased to $48.94 \%(\mathrm{P}<0.001)$, while the infection rates did not differ significantly $(\mathrm{P}=0.581)$. This multi-center study suggests that the Action Plan launched by China's government proved effective in state-owned medical institutions in promoting rational antimicrobial prescription.
\end{abstract}

Keywords: Antimicrobial Use, Multi-center Study, Action Plan

\section{Introduction}

Resistance to antimicrobial agent is a natural biological phenomenon that is becoming increasingly apparent [1-2]. Antimicrobial resistance (AMR) refers to resistance of a microorganism (bacterium, virus, fungus, or parasite) to an antimicrobial agent to which it was previously sensitive. AMR has been elevated to international focus in terms of patient safety and financial burdens incurred [3]. Irrational antimicrobial usage is common and may take the forms of inappropriate prescribing and administering in developed as well as developing countries, especially in China [4-5]. Furthermore, the rapidity with which antimicrobial resistance emerges and its extent are proportional to the density of antimicrobial use [6].

The emergence and development of AMR are a multifaceted issue driven by many interconnected socio-technological factors, including (1) lack of knowledge on differential diagnoses, infectious diseases and microbiology about the appropriate choice of antimicrobial therapies, resulting in inappropriate prescribing practice, as demonstrated in one study in China, in which $63 \%$ of antimicrobials selected to treat proven bacterial infections were found to be inappropriate [4]; (2) lack of knowledge on antimicrobial prophylaxis, which is a common reason for excessive prescribing in many hospitals, as revealed in studies 
in which patients benefited from those procedures and those in which they did not [7-8]; (3) various patient factors, including lack of knowledge on illness and medicine, self-medication, poor adherence to antimicrobial regimen [9-10]; and (4) distorted financial incentives for prescribing antimicrobials, as observed in a previous study in which drug sales formed part of health worker salaries, resulting in consequent greater polypharmacy [11].

Due to the complexity of AMR, a global and national comprehensive response is urgently needed to contain the growing threat of AMR. National government and healthcare systems can have considerable impact on limiting the emergence and development of AMR through the introduction of legislations and regulations concerning the development, licensing, distribution, and clinical application of antimicrobial agents. Actions taken at the national level play critical roles in containing AMR as advocated by the WHO Global Strategy for Containment of Antimicrobial Resistance [12], and actions at national level have been taken to tackle this global threat worldwide [13-15]. On July 1, 2011, the National Antimicrobial Action Plan (referred to as Action Plan thereafter) went into effect in state-owned Grade 2 and 3 hospitals (the top two grades according to Chinese hospital grading system [16]) across China [17].

In this retrospective qualitative and quantitative study, the impacts of the Action Plan on promoting rational antimicrobial usage were evaluated by analyzing the medical records in 28 state-owned hospitals (Grade 2 and 3) across China.

\section{Methods}

\subsection{Practice Settings and Subjects}

Before the introduction of the Action Plan, the practice patterns of state-owned hospitals were also under constant regulations from healthcare authorities ranging from statutes affecting the overall patterns of healthcare delivery to guidelines for medication usage. The regulations that played roles in the overall healthcare provision included (1) the Regulations on Pharmacy Administration in Medical Institutions [18], in which the establishment of drug and therapeutics committee (DTC) was mandated and terms of reference were clarified; (2) the National Essential Medicine List (EML) [19], in which essential medicines covered by healthcare insurance policies were defined; (3) the National Formulary [20], which served as standard treatment guidelines (STGs) for clinicians in deciding on the appropriate treatment for individual patients; (4) the Principles for Clinical Antimicrobial Usage [21], which outlined antimicrobial usage for the purposes of antimicrobial therapy and prophylaxis; (5) the Guidelines for Perioperative Prophylactic Treatment [22], by which surgical procedures were categorized into four classes as clean, clean-contaminated, contaminated, and dirty surgical procedures and the principles for antimicrobial prophylaxis were specified; and (6) the Principles for Perioperative
Prophylactic Treatment in Clean Surgeries [23], which covered information on indication for prophylaxis, choice of agent, timing of administration, and clinical considerations specific to clean surgical procedures.

However, various problems did exist before the introduction of the Action Plan concerning medication usage on many different levels: (1) the chief executive of a hospital was required to assume responsibility for the clinical outcome from antimicrobial treatment, as defined in the terms of reference of drugs and therapeutic committee [18]. However, antimicrobial usage was not placed high on the agenda in many hospitals; (2) fully-functioning infectious disease department and microbiology department were not prioritized in many hospital partly due to inadequate realization of the significance of the two departments in containing AMR; (3) structured antimicrobial management system was absent, resulting in unlimited antimicrobial prescribing by clinicians; (4) many hospitals maintained a stock of antimicrobials exceeding 100 classes; (5) a comprehensive national level antimicrobial usage monitoring system was not existent, failing to providing the needed statistics which could be the knowledge base for public health surveillance.

Inspired by precious studies in probing the effects of certain interventions on public health [24-25], this multi-center cohort study was initiated in November 2011, covering 28 state-owned Chinese hospitals from 14 provinces or municipalities. The medical records in investigation were divided into 2 periods: the baseline period, 3 months, from October 1, to December 31, 2010; and the intervention period, 3 months, from October 1 to December 31, 2011. For reference purposes, the medical data between the baseline and the intervention periods were also analyzed aiming at providing a whole picture. The medical data in this study covered 14,431,923 hospital admissions, among which $13,563,365$ cases were from outpatient setting and 868,558 cases were from inpatient setting.

\subsection{Interventions Introduced by the Action Plan}

At the beginning of intervention period, the Ministry of Health launched the Action Plan targeting irrational antimicrobial usage, mandating compulsory participation from Grade 2 and 3 state-owned hospitals. The Action Plan mandated that: (1) the chief executive of the hospital be responsible for the quality and cost-effectiveness of antimicrobial usage and for the implementation of the regulations and guidelinestargeting irrational antimicrobial usage across the institution on any aspect [21-23]; (2) infectious disease department and microbiology department be established and strengthened to work in collaboration with other clinicians with the responsibilities of the daily management of infection control; (3) structured level-of-use (non-restricted, restricted, and very-restricted) antimicrobial policies be established and clinicians be trained and appraised before being authorized the level-of-use antimicrobial prescribing or dispensing qualification; (4) antimicrobial prophylaxis be administered 2 hours before incision and a 
single dose of selected antimicrobial be administered for patients receiving clean surgical procedures in most cases; and (5) regulations on antimicrobial procurement and supply be enforced.

The Action Plan also focused on surveillance and infection control. Microbiologists and infection control specialists were responsible for the surveillance of pathogen susceptibility patterns and at both departmental and institutional levels and for disseminating the information or knowledge acquired to all departments. Furthermore, the prevalence of local pathogens and their susceptibilities to antimicrobial agents were required to report to a national AMR surveillance program [26].

Aiming at limiting the overall antimicrobial uses, the Action Plan set specific goals to achieve: (1) the proportion of patients receiving antimicrobial treatment should not exceed $20 \%$ and $60 \%$ in outpatient and inpatient settings, respectively; (2) antimicrobial consumption should be controlled below 40 DDD for inpatient; (3) the proportion of antimicrobial prophylaxis for inpatients undergoing clean surgical procedures should not exceed $30 \%$; (4) the stock of antimicrobial classes should be kept below 50 in Grade 3 hospitals or 30 in Grade 2 hospitals; and (5) restrictions were placed to the routine stock of major antimicrobials including third-generation and fourth-generation cephalosporins, carbapenems, fluoroquinolones, and antifungals for deep-seated mycosis.

\subsection{Outcome Measures}

The primary outcome measures were changes in antimicrobial prescribing, which were further divided into two sub-indicators. First, the changes in the proportion of patients receiving antimicrobial treatment were assessed in both outpatient and inpatient settings by analyzing medical data for the baseline and intervention periods, respectively. Second, the changes in the intensity of antimicrobial prescription were investigated in both outpatient and inpatient settings. The measurements of intensity were presented as DDD for inpatients and DDDs (defined daily doses per thousand patients per day) for outpatients.

The secondary outcome measures were changes in the expenses during hospital stay in inpatient setting between the baseline and intervention periods, which were further divided into four sub-indicators: (1) the total expenses during hospital stay; (2) expenses on medicines; (3) expenses on antimicrobials; and (4) expenses on very-restricted antimicrobials.

The tertiary outcome measure was changes in prescribing antimicrobial prophylaxis and related surgical site infection (SSI) rates in clean surgical procedures. This prescribing indicator reflected the impacts of the Action Plan on prescribing prophylaxis by making comparison between patients receiving antimicrobial prophylaxis for clean surgical procedures during the baseline and intervention periods.

\subsection{Statistical Analyses}

The statistics were analyzed using the statistical software SPSS version 19.0. Statistics were represented in different manners depending on their distribution patterns revealed by One-Sample Kolmogorov-Smirnov test. For the data following normal distribution, statistics were expressed as means \pm SD followed by Student's t-test or corrected t-test. Otherwise, the data were expressed by medians plus $95 \%$ confidence intervals followed by Wilcoxon rank sum test. All statistical tests were two-sided and $\mathrm{P} \leq 0.05$ was considered statistically significant.

\section{Results}

Preliminary Kolmogorov-Smirnov tests revealed that the statistics investigated herein followed normal distribution except for two indices which were the percentage of inpatients receiving antimicrobial prophylaxis for clean surgical procedures and related SSI rates.

\subsection{The Action Plan Facilitated Rational Antimicrobial Prescribing}

Compared to the baseline period, the percentage of patients receiving antimicrobials in the intervention period decreased from $26.6 \%$ to $15.6 \%$ in outpatient setting and from $64.8 \%$ to $49.1 \%$ in inpatient setting, respectively (Figure 1, Table 1).

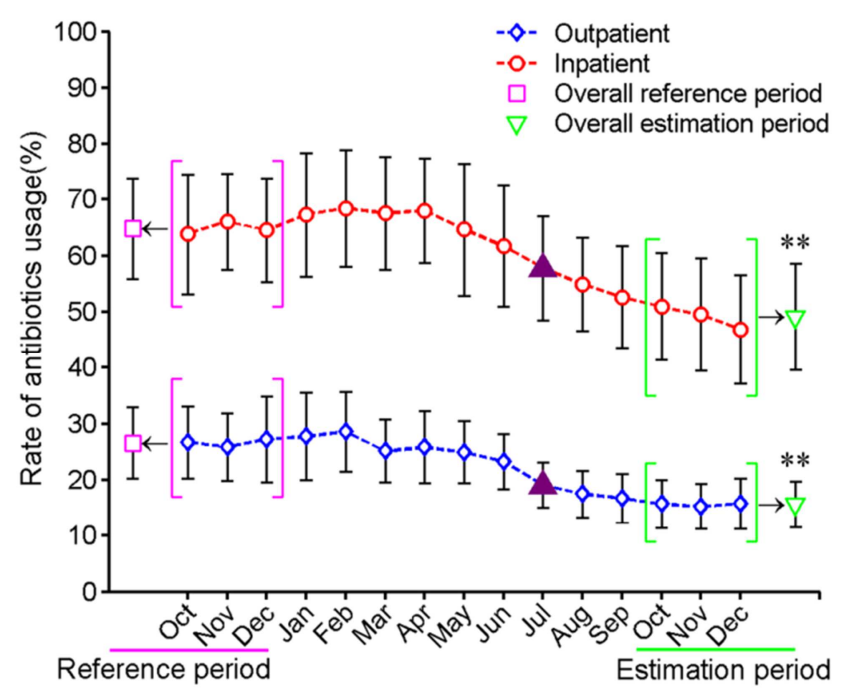

Figure 1. Inpatient and outpatient use rates of antimicrobials before and after "Action Plan". The overall use rates of antimicrobials in the reference period and the estimation period differ significantly $\left({ }^{*} P<0.001\right)$.

Meanwhile, the intensity of antimicrobials prescribed decreased from 69.44 to 50.29 DDD in inpatient setting, while the DDDs per thousand patients per day dropped from 16632.31 to 12914.33 DDDs in outpatient setting (Figure 2, Table 1). 
A

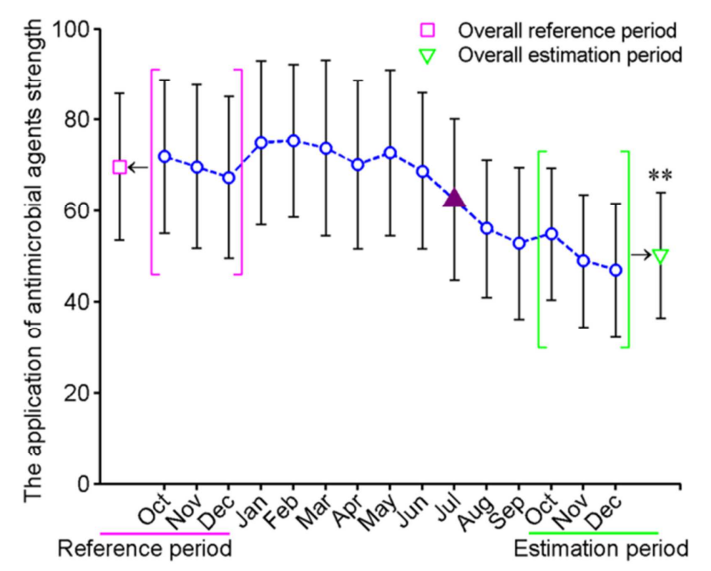

B

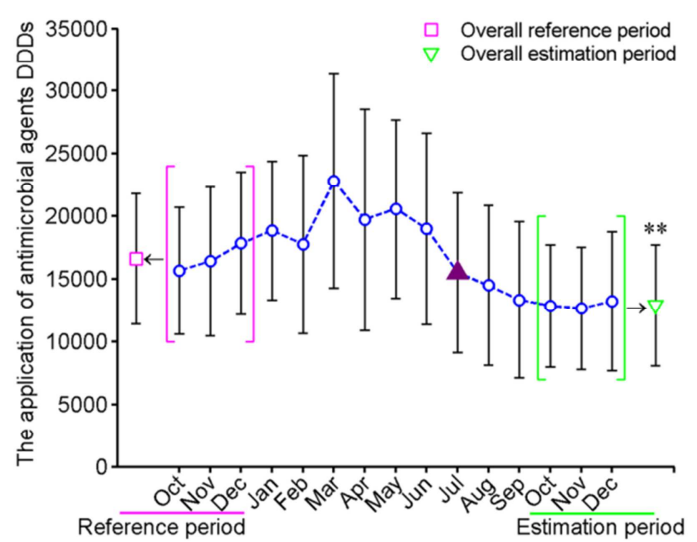

Figure 2. Inpatient use densities of antimicrobials (A) and monthly outpatient consumption of antimicrobials (B) before and after the Action Plan. The overall data in the reference period and the estimation period differ significantly $\left({ }^{* *} P<0.001\right)$.

\subsection{The Action Plan Reduced Expenses on Antimicrobial Agents in Inpatient Setting}

Compared to the baseline period, the total expenses during hospital stay (Figure 3A, Table 1) and expenses on medicines (Figure 3B, Table 1) in the intervention period did not change

A

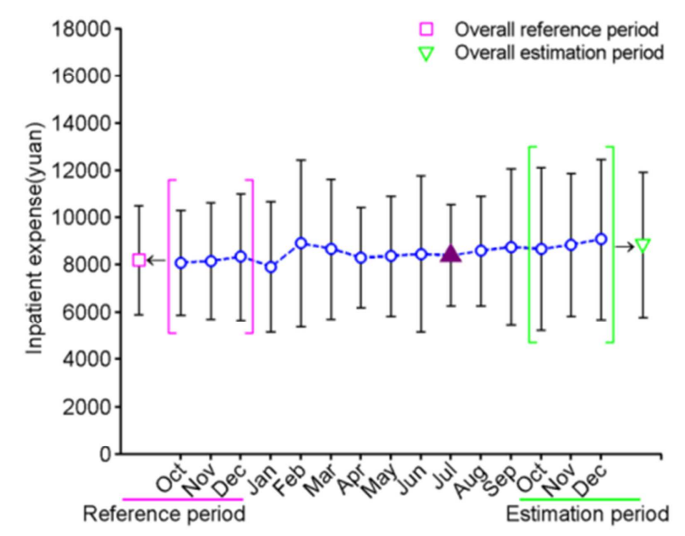

C

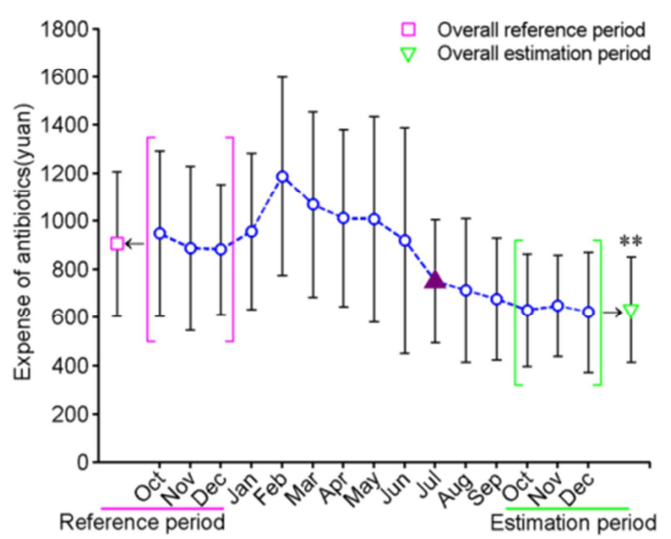

significantly $(P=0.828)$. By contrast, the expenses on antimicrobials (Figure 3C, Table 1) and on special antimicrobials (Figure 3D, Table 1) significantly decreased from 906.58 CNY (China Yuan) to $633.34 \mathrm{CNY}$ and from $321.18 \mathrm{CNY}$ to $145.42 \mathrm{CNY}$, respectively $(P<0.001)$.

B

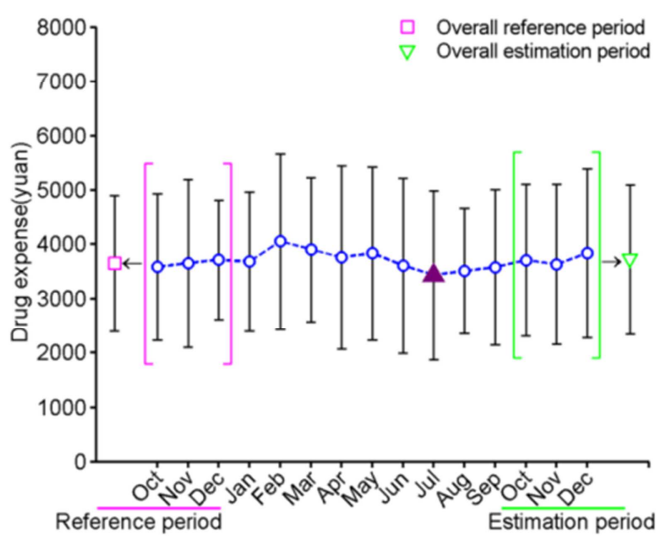

D

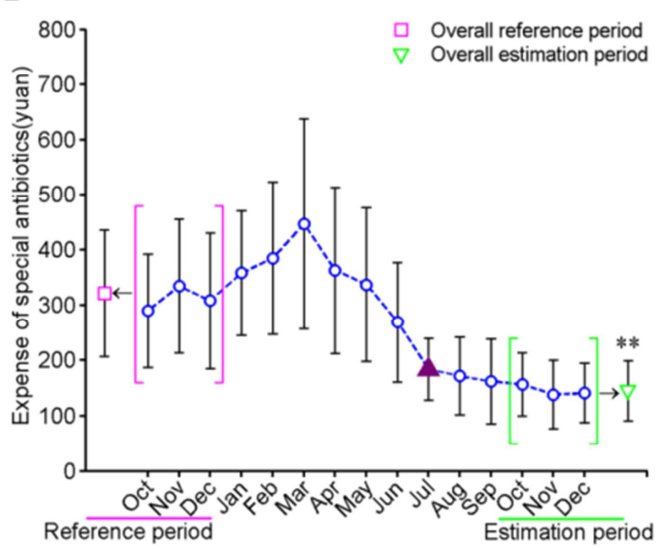

Figure 3. Economic indexes (A: average hospitalized charge; $B$ : average drug expense of inpatients; C: average antimicrobial charge of inpatients; D: average special agent charge of inpatients) before and after "Action Plan". The overall data in the reference period and the estimation period differ significantly $\left({ }^{* *} P<0.001\right)$. 
Table 1. Use of antimicrobials before and after the Action Plan $(\bar{x} \pm s)$.

\begin{tabular}{lllll}
\hline Index & Reference period $(\mathbf{n}=\mathbf{2 8})$ & Estimation period $(\mathbf{n}=\mathbf{2 8})$ & $\mathbf{t}$-Value & P-Value \\
\hline AURO & $26.60 \pm 6.32$ & $15.59 \pm 4.07$ & $7.892^{\mathrm{a}}$ & $<0.001$ \\
AURI & $64.84 \pm 8.98$ & $49.11 \pm 9.43$ & $6.506^{\mathrm{a}}$ & $<0.001$ \\
AADDDI & $69.64 \pm 16.13$ & $50.29 \pm 13.75$ & $4.917^{\mathrm{a}}$ & $<0.001$ \\
ADDDO & $16632.31 \pm 5188.94$ & $12914.33 \pm 4819.02$ & $2.817^{\mathrm{a}}$ & 0.007 \\
AHC & $8193.37 \pm 2288.31$ & $8852.88 \pm 3066.31$ & $0.928^{\mathrm{a}}$ & 0.357 \\
ADEI & $3649.98 \pm 1255.27$ & $3725.68 \pm 1379.84$ & $0.219^{\mathrm{a}}$ & 0.828 \\
AACI & $906.58 \pm 300.21$ & $633.34 \pm 218.61$ & $3.962^{\mathrm{a}}$ & $<0.001$ \\
ASACI & $321.18 \pm 114.72$ & $145.42 \pm 53.76$ & $7.471^{\mathrm{b}}$ & $<0.001$ \\
\hline
\end{tabular}

a: Student's t test; b: Corrected t-test

AURI: antimicrobial use rate of inpatients; AURO: antimicrobial use rate of outpatients; AADDDI: antimicrobial agent defined daily dose of inpatients; ADDDO: antimicrobial DDD of outpatients; ADDDOP: antimicrobial DDD of outpatient per thousand population; AACI: average antimicrobial charge of inpatients; ADEI: average drug expense of inpatients; AHC: average hospitalized charge; ASACI: average special agent charge of inpatients

\subsection{The Action Plan Improved Rational Antimicrobial Prophylaxis}

Since the percentage of inpatients receiving antimicrobial prophylaxis for clean surgical procedures and related SSI rates in both the baseline and intervention periods did not follow normal distribution, statistics were expressed as medians plus 95\% confidence intervals. Compared to the baseline period, the percentage of antimicrobial prophylaxis for clean surgical procedures significantly decreased from $73.6 \%$ to $48.9 \%$ $(P<0.001)$, while the SSI rates did not change significantly $(P=0.581)$, as was shown in Table 2 .

Table 2. Use rates of antimicrobials and infection rates in clean surgical procedures. [Median ( $Q L \sim Q u)]$.

\begin{tabular}{|c|c|c|c|c|}
\hline & Reference period $(95 \%$ CI) & Estimation period $(95 \% \mathrm{CI})$ & $Z$ value & P value \\
\hline Use rate of antimicrobials (\%) & $73.56(64.52 \sim 78.50)$ & $48.94(41.96 \sim 57.64)$ & -6.539 & $<0.001$ \\
\hline Infection rate $(\%)$ & $0.86(0.652 \sim 0.994)$ & $0.84(0.680 \sim 0.989)$ & -0.553 & 0.581 \\
\hline
\end{tabular}

\section{Discussion}

This research was initiated by the first author, who conceived the design protocol and advocated the research on a pharmacist community well-known to peers by posting research protocols and terms of confidentiality on the website (http://www.clinphar.cn/). Of all the statistics received from 80 hospitals with prior approval from the leadership of the hospitals involved, 28 hospitals were included in the research, while 51 hospitals were excluded due to incompleteness of medical records targeted by this research. The detailed study design and writing of the manuscript were discussed repeatedly by all authors using a popular network meeting software.

Antimicrobials use has been paid particular attention by China's government, aiming at providing secure, effective, convenient, and affordable medical services for patients. It has been previously reported that government intervention directly and significantly influenced medical services [27-29]. Similarly, the gradually rational use of antimicrobials in China results from the intervention of the Chinese Ministry of Health and the established technological supporting system of antimicrobials clinical use. According to relevant policies, departments of infectious diseases and clinical microbiology are required in Grade 2 and 3 hospitals. Professional infectious disease doctors, professional microbiological examination personnel and clinical pharmacists are also required to guide the clinical use of antimicrobials technically and to provide professional training the concerning the clinical use of antimicrobials for physicians. Doctors are permitted to prescribe with different authorities after they have been trained and have passed the corresponding tests. Medical institutions and doctors are strictly restricted in the clinical use of antimicrobials. In principle, no more than 50 and 35 types of antimicrobials are allowed in Grade 2 and 3 hospitals [21, 30].

In this study, changes in prescribing of antimicrobials were quantitatively measured and the impacts were reflected from the indicators of proportion of patients receiving antimicrobial treatment and intensity of antimicrobial therapy and prophylaxis, respectively, while the administration of antimicrobials were the responsibilities of nursing personnel, which was beyond the scope of this article. The average use rates of inpatients and outpatients are $50.3 \%$ and $15.6 \%$, which are both lower than those required by the Action Plan (60\% and $20 \%)$.

Besides, antimicrobials use intensity (AUI) is expressed as the average defined daily dose (DDD) per hundred beds (DDD/100 patients per day) that equals (DDDs/total time of medication for all the patients) $\times 100$, which has been widely used in estimating the extensity and density of antimicrobials for inpatients [31]. Although the average AUI obtained in this study (57.29 DDD) is higher than that required by the Chinese Ministry of Health (40 DDD), it has significantly reduced compared to that obtained in the reference period. Besides, DDDs also significantly decreased.

Moreover, no more than $30 \%$ of the patients enrolled in our study received prophylactic antimicrobials in Class I clean surgical procedures, which is much lower than that in the 
survey of the Health Ministry (96.9\%). General aseptic incision operations, such as surface operations of heads, necks, bodies and limbs, inguinal hernia hernioplasty without artificial implants, thyroid adenoma resections and breast fibroadenoma resections, could be performed without antimicrobials in most cases. Decreasing the use rate of antimicrobials from $73.6 \%$ to $48.9 \%$ in clean surgical procedures did not increase the infection rate, suggesting major clean surgical procedures are not in need of antimicrobials. Accordingly, the Action Plan is able to directly intervene in the prophylactic use of antimicrobials in clean surgical procedures [22].

Though our study provides critical information about antimicrobial intervention, there are some limitations. The Action Plan was considered the most stringent legislation on antimicrobial administration in China's history. Firstly, Task forces (membership including healthcare professionals, veterinarians, pharmaceutical manufacturers, and other interest parties) were created at national, provincial, and local levels in developing, implementing, and monitoring the antimicrobial management across China. This research was conducted in state-owned healthcare institutions. Secondly, all the statistics presented in this study were collected from computerized hospital information systems in respective hospitals, since manual collection of medical statistics was impractical in this non-sponsored research. As a functioning information system represents improved quality and cost-effectiveness in healthcare provision, it is not logical to extrapolate the improved outcome observed in the 28 hospitals to those hospitals without a computerized information system. Thirdly, it was impractical to assign patients at random to two groups or to maintain a control group in this research. Therefore, this study was quasi-experimental [32-34]. The impacts of the interventions brought by the Action Plan were measured as an integrated intervention. Further studies need to be conducted to investigate the impacts of a specific measure as an independent variable.

\section{Conclusion}

It is a long way to strengthen the clinical use management of antimicrobials, boost rational drug use and ensure medical security, which are in need of continuously improved management systems and execution methods. Further development of the Action Plan in China and the resulting management, indexes and working systems will eventually promote the rational use of antimicrobials in China and even all around the world.

\section{Acknowledgements}

This research is funded by the following projects: the Major scientific research projects of Affiliated Hospital of Inner Mongolia Medical University (NYFY ZD 2012017); the Nature Science Foundation of Inner Mongolia Autonomous Region (2017MS0831) for Ruilian Ma, http://nsbr.nmkjt.gov.cn/index.aspx.

\section{References}

[1] Ashley D, Brindle M. Penicillin resistance in staphylococci isolated in a casualty department. J Clin Pathol. 1960; 13: 336-338.

[2] Standing Medical Advisory Committee Sub-Group on Antimicrobial Resistance. 1998. The path of least resistance. London: Department of Health. http://www.dh.gov.uk/en/Publicationsandstatistics/Publication s/PublicationsPolicyAndGuidance/DH_4009357.

[3] World Health Organization. 2011. Antimicrobial resistance. Fact Sheet N 194.

http://www.who.int/mediacentre/factsheets/fs194/en/index.ht $\mathrm{ml}$

[4] Hui L, Li XS, Zeng XJ, Dai YH, Foy HM. Patterns and determinants of use of antimicrobials for acute respiratory tract infection in children in China. Paediatr Infect Dis J. 1997; 16: 560-564.

[5] Xiao YH, Giske CG, Wei ZQ, Shen P, Heddini A, Li LJ. Epidemiology and characteristics of antimicrobial resistance in China. Drug Resist Update. 2011; 14: 236-250.

[6] Alonso R, Padilla B, Sánchez-Carrillo C, Muñoz P, Rodríguez-Creixems M, Bouza E. Outbreak among HIV-infected patients of Staphylococcus aureus resistant to cotrimoxazole and methicillin. Infect Control Hosp Epidemiol. 1997; 18: 617-621.

[7] Woods RK, Dellinger EP. Current guidelines for antimicrobial prophylaxis of surgical wounds. Am Fam Physician. 1998; 57: 2731-2740.

[8] Swedish-Norwegian Consensus Group. Antimicrobial prophylaxis in surgery: summary of a Swedish-Norwegian Consensus Conference. Scand J Infect Dis. 1998; 30: 547 557.

[9] Nichter M. Pharmaceuticals, health commodification and social relations: ramifications for primary health care, Anthropology and International Health, South Asian Case Studies, Kluwer Academic Publishers; 1989. Section 3, No. 9: 233-277.

[10] Branthwaite A, Pechere JC. Pan-European survey of patients' attitudes to antimicrobials and antimicrobial use. J Intern Med Res. 1996; 24: 229-238.

[11] Zhan SK, Tang SL, Guo YD, Bloom G. Drug prescribing in rural health facilities in China: implications for service quality and cost. Trop Doct. 1998; 28: 42-48.

[12] Geneva: World Health Organization. 2001. Global strategy for the containment of antimicrobial resistance. http://www.who.int/csr/resources/publications/drugresist/WH O_CDS_CSR_DRS_2001_2_EN/en/

[13] Center for Disease Control and Prevention. 2001. US Public Health Action Plan to Combat Antimicrobial Resistance (Part 1: Domestic Issues). http://www.cdc.gov/drugresistance/actionplan/actionplan.html

[14] Health Canada. Controlling Antimicrobial Resistance: An Integrated Action Plan for Canadians. Can Comm Dis Rep (CCDR). 1997; Suppl 23S7:1-32. 
[15] European Commission DGXXIV. 1999. Opinion of the Scientific Steering Committee on Antimicrobial Resistance. http://ec.europa.eu/food/fs/sc/ssc/out50_en.pdf

[16] Ma XW. 2011. Video Conference on "National Antimicrobial Action Plan".

http://www.moh.gov.cn/publicfiles/business/htmlfiles/mohzcf gs/s7857/201106/52208.htm

[17] State Council of the People's Republic of China. 2011. National Antimicrobial Action Plan (trial, in Chinese). State Council Document 2011(75) http://bmyj.chinalaw.gov.cn/lismsPro/law_download/fulltext/1 312360890480.doc

[18] Ministry of Health. 2011. Regulation of pharmaceutical affairs administration in medical institutions (In Chinese). http://www.satcm.gov.cn/web2010/zhengwugongkai/zhengcef agui/falvfagui/guizhang/2011-04-07/13297.html

[19] Ministry of Health. 2009. National Essential Medicine List (In Chinese).

http://www.gov.cn/gzdt/2009-08/18/content_1395524.htm.

[20] Ministry of Health. Chinese National Formulary (In Chinese). Beijing: People's Military Medical Press; 2010.

[21] Chinese Medical Association, Pharmacy Professional Advisory Committee of Chinese Hospital Association, Hospital Pharmacy Advisory Committee of Chinese Pharmaceutical Association. Guiding Principles for Clinical Application of Antimicrobials. Adver Drug React J. 2005; 7: 42.

[22] Surgery Branch of Chinese Academy of Medical Sciences, Editorial Board of Chinese Journal of Surgery. Guideline for Perioperative Antimicrobial Prophylaxis. Chin J Surg. 2006; 44: 1594.

[23] Ministry of Health. 2009. Principles for perioperative prophylactic treatment in clean surgical procedures (In Chinese).

http://www.moh.gov.cn/publicfiles/business/cmsresources/mo hylfwjgs/cmsrsdocument/doc6647.doc

[24] Bates DW, Spell N, Cullen DJ, Burdick E, Laird N, Petersen LA, Small SD, Sweitzer BJ, Leape LL. The costs of adverse drug events in hospitalized patients. JAMA. 1997; 227: 307311

[25] Bates DW, Leape LL, Cullen DJ, Laird N, Petersen LA, Teich
JM, Burdick E, Hickey M, Kleefield S, Shea B, Vander Vliet M, Seger DL. Effect of computerized physician order entry and a team intervention on prevention of serious medication errors. JAMA. 1998; 280: 1311-1316.

[26] Ministry of Health. 2010. National Antimicrobial Surveillance System (In Chinese). http://www.chinadtc.org.cn/index.php?option=com_content\& module $=24 \&$ sortid $=0 \&$ artid $=0 \&$ menuid $=35$

[27] Allender S, Gleeson E, Crammond B, Sacks G, Lawrence M, Peeters A, Loff B, Swinburn B. Policy change to create supportive environments for physical activity and healthy eating: which options are the most realistic for local government? Health Promot Int. 2012; 27: 261-274.

[28] Fobil JN, Soyiri IN. An assessment of government policy response to HIV/AIDS in Ghana. SAHARA J. 2006; 3: 457-465.

[29] Bowen S, Zwi A, Sainsbury P. What evidence informs government population health policy? Lessons from early childhood intervention policy in Australia. $N S W$ Public Health Bull. 2005; 16: 180-184.

[30] Ministry of Health of the People's Republic of China. 2011. Action Plan for the Clinical Use of antimicrobials in 2011. http://www.moh.gov.cn/publicfiles/business/htmlfiles/mohyzs/ s3578/201104/51283.htm

[31] Oishi T, Hitomi S, Kamoshita M, Fukue H, Kawahata D, Fukutake K. [Comparison of antimicrobial use density (AUD) of carbapenem antimicrobial agents and investigation of the drug susceptibility of Pseudomonas aeruginosa in 3 hospitals in southern Ibaraki Prefecture, Japan]. Rinsho Byori. 2008; 56: 570-576.

[32] Campbell DT, Stanley JC. Experimental and quasi-experimental designs for research. Chicago, IL: Rand-McNally; 1963.

[33] Cook TD, Campbell DT. Quasi-experimentation: Design and analysis issues for field settings. Boston, MA: Houghton Mifflin Company; 1979.

[34] Varkevisser CM, Pathmanathan I, Brownlee A. Designing and conducting health systems research. Health Systems Research Training Series. Vol. 2 Part 1. Ottawa: International Development Research Centre; 1991. 